\section{Bau und Biologie des fossilen Fisches}

Die Fisch-Art mit dem wissenschaftlichen $\mathrm{Na}$ men Gobius francofurtanus KOKEN 1891 aus der Familie Gobiidae (Meeresgrundeln) tritt im Rhein-Main-Gebiet erstmals in der FrankfurtFormation auf. Dorthin ist sie während einer letzten marin-brackischen Ingression aus der Parathethys über den Oberrheinischen Graben zugewandert. Die Parathethys war ein Arm der Thethys etwa im heutigen westlichen Donauraum; die Thethys war ein sich damals bis nach Ostasien hin erstreckendes Meer, das heutige Mittelmeer ist ein kleiner Rest davon. Das Klima war während des gesamten Miozäns subtropisch.

Grundeln sind eine Untergruppe der barschartigen Fische (Perciformes). Sie haben einen langgestreckten, zylinderförmigen Körper, einen bulligen Kopf mit nach oben stehenden Augen und ein großes Maul. Die stachelstrahlige und die weichstrahlige Rückenflosse liegen deutlich voneinander getrennt. Die Bauchflossen sind in der Regel zu einer trichterförmigen Saugscheibe zusammengewachsen, mit der sich die Grundeln an harten Oberflächen, zum Beispiel an Felsen, anheften können. Diese Lebensweise ist beispielsweise im Exotarium des Frankfurter Zoos bei heute lebenden Grundeln gut zu beobachten. Die meisten Arten haben keine Schwimmblase, weshalb sie sich häufig auf dem Boden oder auf Felsen aufhalten.

\section{Literatur}

Oтт, W. 2003: Baustellen: Fenster in die Erdgeschichte. 3. Mitteilung. Neubau der U-Bahnstation Senckenberganlage, Frankfurt am Main-Bockenheim. Eine ergiebige Fundstelle fossiler Fischskelette in den Oberen HydrobienSchichten. - Ber. Offb. Ver. Naturkde. 103: 28-45.

\section{Internetseite}

http://www.palaeo-geo-ev.de/Fossiliengalerie.htm

(mehrere Abbildungen von Gobius von dem in unmittelbarer Nähe zur hier beschriebenen Fundstelle liegenden

Baustellenaufschluss Senckenberganlage)

\title{
Botanisch-gärtnerische Literatur
}

\section{MARTIN BÖRNCHEN}

Farben bereichern unser Leben. Tierische und pflanzliche Farbstoffe. Ausstellungsführer der Universitätsbibliothek der Freien Universität Berlin, Bd. 48.

Universitätsbibliothek der Freien Universität Berlin, Berlin 2009, 60 S., 142 farbige Abbildungen, 29 x 21 cm, Paperback, ISBN 978-3-929619-52-2;10 € zzgl. $2 €$ Versandkosten

Dass sich MARTIN BÖRNCHEN seit Jahren mit Safran und anderen Naturfarbstoffen beschäftigt, zeigt sich an dem sehr ansprechenden Ausstellungskatalog, der als Ergänzung zur Ausstellung in der Berliner Universitätsbibliothek entstanden ist. Nach einer ganz kurzen Einführung über Licht und Farbe widmet sich der Katalog den Farbstofflieferanten Purpurschnecke, Kermes- und Cochenille-Laus, Krapp, Safran, Färberdistel, Färberwaid, Indigostrauch und Gallapfel. Im letzten Kapitel „PERKINs Mauvein“ wird ein Ausblick auf die Anilinfarben gegeben, die schließlich die natürlichen Pflanzenfarbstoffe verdrängten.

Purpur war in der Antike der teuerste Farbstoff. Anhand eindrucksvoller Fotos werden Purpurschnecke und die Verwendung ihres Drüsenfarbstoffes dargestellt. MARTIN
BÖRNCHEN lässt es sich nicht nehmen, auf die Schwierigkeiten bei Recherchen und der Materialbeschaffung für die Ausstellung hinzuweisen. So zitiert er einen an ihn gerichteten Brief der Fotografin YaSmine EID-SABBAGH, die nur unter militärischer Begleitung den sogenannten Murex-Hill im antiken Sideon aufsuchen und fotografieren konnte und dabei nur eine Müllhalde vorfand.

Auch die nachfolgenden Kapitel liefern klar verständliche und sehr detaillierte Informationen. Die Zusammenstellung der Texte und hervorragenden Abbildungen sowie das umfangreiche Quellenverzeichnis am Ende lassen erkennen, dass BÖRNCHEN die Inhalte sehr gut recherchiert hat. Auch den komplizierten Vorgang des Blaufärbens mit Hilfe von Waid weiß er verständlich zu erklären.

Dieser vom Umfang her überschaubare Katalog ist eine gute Ergänzung zum im Palmengarten erschienenen Sonderheft „Farbe in der Natur“, da hier neben einzelnen Färbepflanzen auch Farbstoff liefernde Tiere ausführlicher abgehandelt werden. Der Katalog spricht diejenigen an, die sich für Naturfarben, insbesondere deren Chemismus und Kulturgeschichte, interessieren.

HILKE STEINECKE 